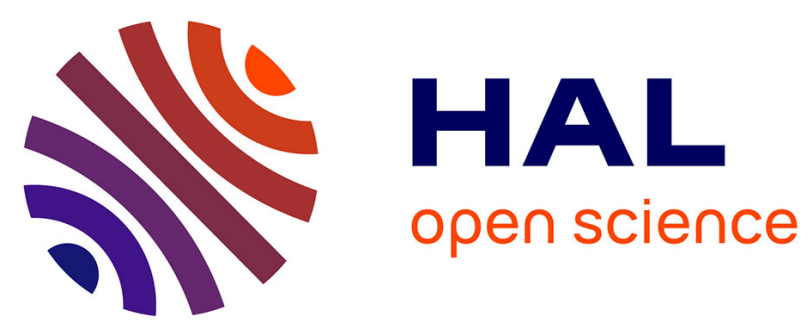

\title{
Decreased RNF41 expression leads to insulin resistance in skeletal muscle of obese women
}

Cyril Breuker, Cacylde Amouzou, Odile Fabre, Karen Lambert, Pascal Seyer, Annick Bourret, Tamim Salehzada, Jacques Mercier, Ariane Sultan, Catherine Bisbal

\section{To cite this version:}

Cyril Breuker, Cacylde Amouzou, Odile Fabre, Karen Lambert, Pascal Seyer, et al.. Decreased RNF41 expression leads to insulin resistance in skeletal muscle of obese women. Metabolism, 2018, 83, pp.81 - 91. 10.1016/j.metabol.2018.01.014 . hal-01796389

\section{HAL Id: hal-01796389 https://hal.science/hal-01796389}

Submitted on 3 Jan 2020

HAL is a multi-disciplinary open access archive for the deposit and dissemination of scientific research documents, whether they are published or not. The documents may come from teaching and research institutions in France or abroad, or from public or private research centers.
L'archive ouverte pluridisciplinaire HAL, est destinée au dépôt et à la diffusion de documents scientifiques de niveau recherche, publiés ou non, émanant des établissements d'enseignement et de recherche français ou étrangers, des laboratoires publics ou privés. 


\title{
Decreased RNF41 expression leads to insulin resistance in skeletal muscle of obese women
}

\author{
Cyril Breuker ${ }^{\mathrm{a}, \mathrm{b}}$, Cacylde Amouzou ${ }^{\mathrm{a}}$, Odile Fabre ${ }^{\mathrm{a}}$, Karen Lambert ${ }^{\mathrm{a}}$, Pascal Seyer ${ }^{\mathrm{a}}$, Annick Bourret ${ }^{\mathrm{a}}$, \\ Tamim Salehzada a , Jacques Mercier ${ }^{\mathrm{a}, \mathrm{b}}$, Ariane Sultan ${ }^{\mathrm{a}, \mathrm{b}}$, Catherine Bisbal ${ }^{\mathrm{a}, *}$ \\ a PhyMedExp, University of Montpellier, INSERM U1046, CNRS UMR 9214, 34295 Montpellier cedex 5, France \\ ${ }^{\mathrm{b}}$ Centre Hospitalier Universitaire (CHU) Montpellier, 34295 Montpellier, France
}

\section{Keywords:}

Obesity

Inflammation

Innate immunity

Interferon

Insulin resistance

Skeletal muscle

\begin{abstract}
A B S T R A C T
Context: Toll-like receptor 4 (TLR4) activation contributes to obesity-associated insulin resistance in skeletal muscles (SM). TLR4 signaling involves two pathways: the myeloid differentiation primary response gene 88 (MyD88) leading to inflammatory cytokines production and the toll/interleukin-1 receptor domain-containing adapter-inducing interferon (IFN) I (TRIF)-dependent pathways leading to type 1 interferon (IFNI) and interferon stimulated genes (ISG) expression. The E3 ubiquitin ligase RNF41 allows the preferential activation of the TRIF-IFNI pathway; however, its role in insulin response has not been reported.

Methods: We measured RNF41 level and IFNI pathway activation (ISG expression) in SM biopsies of obese insulin sensitive (OIS) and obese insulin resistant (OIR) women. Then we isolated and differentiated in myotubes, primary human SM cell progenitors from OIS and OIR SM biopsies. We modulated RNF41 and ISG expression in these myotubes and investigated their effects on insulin response.

Results: RNF41 expression is down-regulated in vivo in OIR SM and myotubes compared to OIS SM and myotubes. TLR4 activation with palmitate induces TRIF-IFNI pathway and ISG in OIS myotubes but not in OIR myotubes. Inhibition of RNF41 expression with siRNF41 in OIS myotubes treated with palmitate attenuates insulin response, IFNI pathway activation and ISG induction, mimicking OIR phenotype. Further, overexpression of RNF41 in OIR myotubes increases insulin response and ISG expression. Exposure to IFNI or to its inducer polyinosinicpolycytidylic acid, restores ISG expression and insulin sensitivity in OIR myotubes and OIS myotubes transfected with siRNF41.

Conclusion: Our results identify RNF41 as essential to IFNI pathway activation in order to maintain muscle insulin sensitivity during human obesity.
\end{abstract}

\section{Introduction}

Obesity is a worldwide epidemic disease and a major risk factor for insulin resistance (IR) development in insulin-dependent organs such as skeletal muscles (SM) [1,2]. Ultimately, IR leads to the onset of type 2 diabetes [3,4]. In fact, SM is responsible for $80 \%$ of glucose uptake

Abbreviations: BSA, bovine serum albumin; IFN, interferon; IR, insulin resistance; IRF3, interferon regulatory factor 3; ISG, interferon-stimulated genes; ISRE interferon-sensitive/stimulated response element; LPS, lipopolysaccharides; MyD88, myeloid differentiation primary response gene 88 ; $\mathrm{NF} \kappa \mathrm{B}$, nuclear factor $\kappa \mathrm{B}$; NEFA, non-esterified fatty acids; OAS, oligoadenylate synthetase; OIS, obese insulinsensitive; OIR, obese insulin-resistant; PolyIC, polyinosinic-polycytidylic acid; SM, skeletal muscle; RNase L, endoribonuclease L; RLI/ABCE1, RNase L inhibitor/ATP binding cassette E1; RNF41, ring finger protein 41; TLR, toll-like receptor; TRIF, toll/interleukin-1 receptor domain-containing adapter-inducing interferon I; 2-5A, 2 '-5' oligoadenylates.

* Corresponding author at: PhyMedExp, University of Montpellier, INSERM U1046, CNRS UMR 9214, 371 avenue du Doyen Gaston Giraud, 34295 Montpellier cedex 5, France.

E-mail address: catherine.bisbal@inserm.fr (C. Bisbal). and metabolism in the postprandial state, and muscle failure in this function is often considered as the first defect causing IR [3,5]. These last years, it has been shown that during obesity, activation of the innate immunity receptor toll-like receptor (TLR) 4 by non-esterified fatty acids (NEFA) and/or lipopolysaccharides (LPS) from the gut microbiota plays a pivotal role in the development of IR [6-9]. However, while the cellular and molecular mechanisms leading from TLR4 activation to IR are still not fully understood in humans, the regulation of its two downstream signaling pathways, the myeloid differentiation primary response gene 88 (MyD88)- and the toll/interleukin-1 receptor domain-containing adapter-inducing interferon (IFN) I/ $/$ (TRIF)-dependent pathways, has never been described in obesity nor in IR. The MyD88 pathway initiates the inflammatory cascade response via nuclear factor $\kappa \mathrm{B}(\mathrm{NF} \kappa \mathrm{B})$ activation and further induction of inflammatory cytokine expression whereas the TRIF pathway leads to interferon regulatory factor 3 (IRF3) activation [10-12]. IRF3 binds the interferon-sensitive/stimulated response element (ISRE) and induces the transcription of IFNI, of interferon stimulated genes (ISG) such as 
the oligoadenylate synthetases (OAS) and of anti-inflammatory factors $[13,14]$. The OAS, themselves activated by double-stranded RNA, synthesize the 2 ' -5 ' oligoadenylates $(2-5 \mathrm{~A})$ which in turn activate the latent endoribonuclease L (RNase L) [15]. RNase L cleaves singlestranded RNA, giving rise to smaller RNA fragments able to activate TLR3 [16]. Of note, TLR3 signaling is, like TLR4, mediated via the TRIF pathway $[10,11]$.

Interestingly, we have previously shown that a defect in RNase L activity leads to IR development in myotubes obtained from obese individuals [17]. Besides, RNase L activation by $2-5 \mathrm{~A}$ transfection enables this defective pathway to reactivate, restoring insulin response in human insulin-resistant myotubes. At the opposite, RNase L inhibition by RNase L inhibitor (RLI)/ATP binding cassette E1 (ABCE1) [18] induces IR in myotubes [17]. These results thus highlight the crucial role of the TRIF-IFNI pathway and particularly the activation of the OAS-RNase L pathway in maintaining insulin sensitivity in human myotubes.

Systemic insulin sensitivity is preserved in some obese individuals who do not display the typical metabolic disorders associated with obesity $[5,19,20]$. Being well aware of the existence of different subgroups of insulin sensitivity levels among obese individuals, we recently compared metabolic parameters and inflammation both at systemic and SM levels in obese insulin-sensitive (OIS) and obese insulinresistant (OIR) post-menopausal women. In accordance with the literature [12], we observed a lower expression of I $\kappa \mathrm{B} \alpha$ in OIR SM compared to OIS SM, indicative of TLR4-MyD88 activation in OIR SM and not in OIS SM [5]. The aim of this study was to better understand the molecular mechanisms underlying IR development in SM during human obesity, in particular the regulation of TRIF-IFNI pathway. In this regard, we focused on RNF41, an E3 ubiquitin ligase ring finger protein 41 (RNF41) identified as an essential positive regulator of TRIF-IFNI pathway at the expense of the MyD88 pathway [21]. We thus compared TRIF-IFNI pathway activation through OAS-RNase L pathway and RNF41 expression in both SM biopsies and biopsy-derived cells from our cohort of OIS and OIR post-menopausal women, previously characterized for their systemic and SM insulin sensitivity [5]. Furthermore, we modulated RNF41 and ISG expression in myotubes differentiated from primary

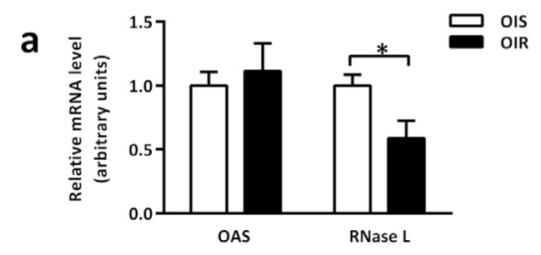

b

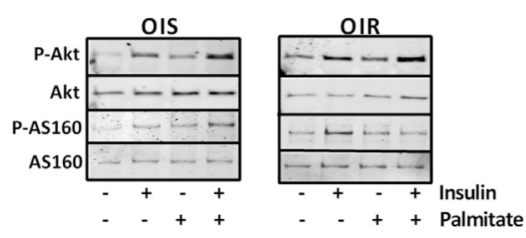

C
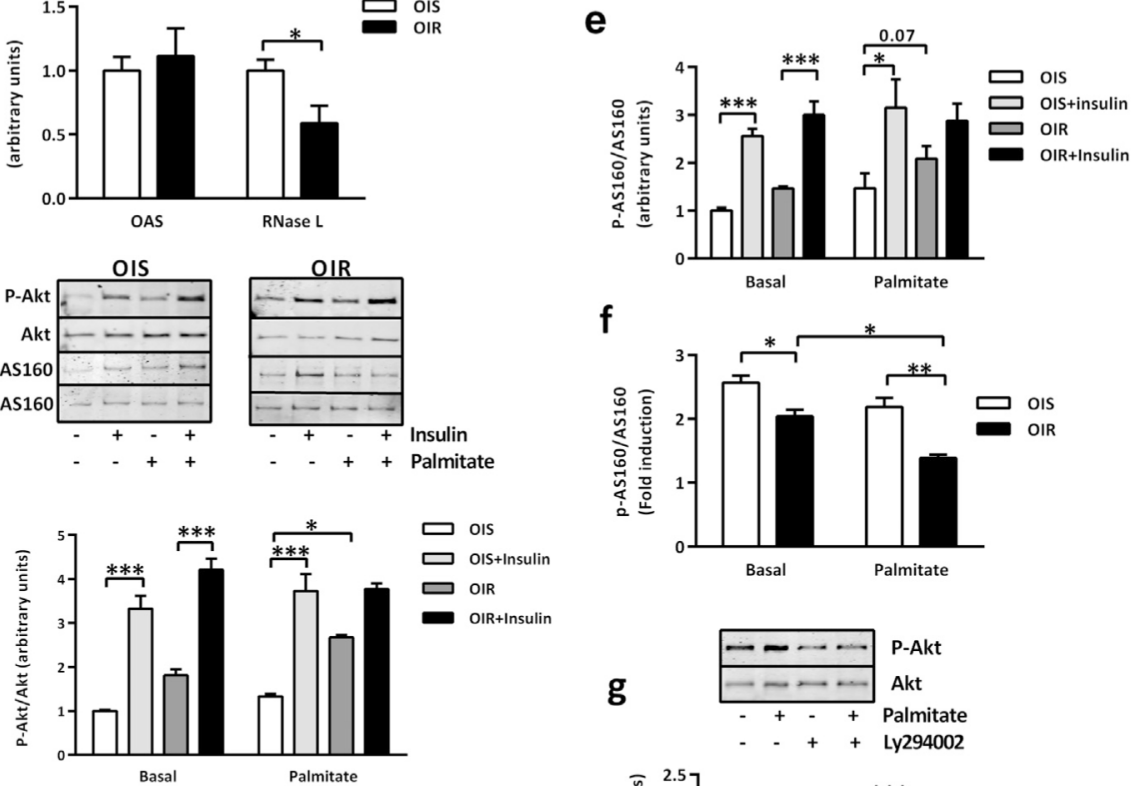

d
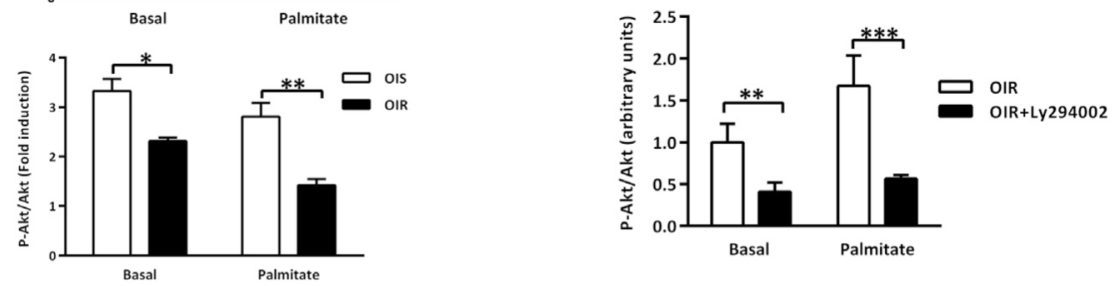

Fig. 1.OAS and RNase L mRNA expression in skeletal muscle and myotubes of OIS and OIR subjects and nsulin response in OIS and OIR myotubes. Panel a: Quantification by qPCR of OAS and RNase $L$ mRNA expression in OIS and OIR SM. Data are expressed as mean \pm SEM and relatively to the mean level of expression in OIS SM, which was set at 1 . Mann-Whitney tests were performed for comparison of OIS and OIR SM biopsies. RNase L mRNA: ${ }^{*} P_{\text {OIS vs oIR }}=0.03$. Panel b: Representative membrane scans for Phospho-Akt (Ser473), Phospho-AS160 (Thr642), Akt and AS160 protein expression are shown for OIS and OIR myotubes treated or not with palmitate and then stimulated or not with insulin. Panel c: Quantification of Phospho-Akt/Akt protein ratio in OIS and OIR myotubes treated or not with palmitate and then stimulated or not with insulin. Data are expressed as mean \pm SEM and relatively to the mean level of Phospho-Akt (Ser473)/Akt ratio in OIS myotubes in basal condition (with neither palmitate treatment nor insulin stimulation), which was set at 1. Error bars refer to the SEM obtained in three independent experiments. Group comparisons were performed using 2 -way ANOVA and Tukey's multiple comparisons test. ${ }^{* * *} P_{O I S}$ vs oIS+ins $={ }^{* * *} P_{O I R}$ vs OIR+ins $={ }^{* * *} P_{\text {OIS Palm vs oIS Palm }+i n s}=0.0003,{ }^{*} P_{\text {OIS Palm vs oIR Palm }}=0.03$. Panel d: Insulin response is determined by the fold induction of Phospho-Akt (Ser473)/Akt protein ratio by insulin stimulation in basal condition or after palmitate treatment. Error bars refer to the SEM obtained in three independent experiments. Group comparisons were performed using 2-way ANOVA and Tukey's multiple comparisons test. ${ }^{*} P_{\text {OIS vs }}$ OIR $=0.03$; ${ }^{* *} P_{\text {OIS Palm vs OIR Palm }}=0.009$. Panels e: Quantification of Phospho-AS160/AS160 protein ratio in OIS and OIR myotubes treated or not with palmitate and then stimulated or not with insulin. Data are expressed as mean \pm SEM and relatively to the mean level of Phospho-AS160 (Thr642)/AS160 ratio in OIS myotubes in basal condition (with neither palmitate treatment nor insulin stimulation), which was set at 1 . Error bars refer to the SEM obtained in three independent experiments. Group comparisons were performed using 2-way ANOVA and Tukey's multiple comparisons test. ${ }^{* * *} P_{\text {OIS vs oIS+ins }}=0.0008,{ }^{* * * *} P_{O I R}$ vs OIR+ins $=0.0007$, ${ }^{*} P_{\text {OIS Palm vs OIS Palm+ins }}=0.03$. Panel f: Insulin response is determined by the fold induction of Phospho-AS160 (Thr642)/AS160 protein ratio by insulin stimulation in basal condition or after palmitate treatment. Error bars refer to the SEM obtained in three independent experiments. Group comparisons were performed using 2-way ANOVA and Tukey's multiple comparisons test. ${ }^{*} P_{\text {OIS vs OIR }}=0.04,{ }^{* *} P_{\text {OIS Palm vs OIR Palm }}=0.006,{ }^{*} P_{\text {OIR vs OIR Palm }}=0.01$. Panel g: Representative membrane scans for Phospho-Akt (Ser473) and Akt protein expression are shown for OIR myotubes treated or not with Ly294002, a known inhibitor of the PI3K, and treated or not with palmitate. Quantification of Phospho-Akt/Akt protein ratio in OIR myotubes. Data are expressed as mean \pm SEM and relatively to the mean level of Phospho-Akt/Akt ratio in OIR myotubes in basal condition (with neither palmitate treatment nor Ly294002), which was set at 1. Error bars refer to the SEM obtained in three independent experiments. Group comparisons were performed using 2-way ANOVA and Tukey's multiple comparisons test. ${ }^{* *} P_{\text {OIR vs } \text { OIR }+ \text { Lyz294002 }}=0.006,{ }^{* * *} P_{\text {OIR Palm vs OIR Palm+Ly294002 }}=0.0003$. 
by Wang et al. [29] with some modifications [17]. Palmitate was dissolved in ethanol to a concentration of $0.75 \mathrm{M}$. This stock solution was diluted extemporaneously in $20 \%$ non-esterified free fatty acid (NEFA)-endotoxin-free bovine serum albumin (BSA) (Sigma-Aldrich) to a working concentration of $7.5 \mathrm{mM}$. This solution was then diluted to $500 \mu \mathrm{M}$ in cell culture media. For insulin response, myotubes were incubated with or without human insulin $0.6 \mu \mathrm{M}$ (Umuline RAPIDE, Lilly, Neuilly-sur-Seine, France) during $10 \mathrm{~min}$ at $37^{\circ} \mathrm{C}$. To confirm that the phosphorylation of Akt was only due to insulin PI3K pathway, cells were treated with LY294002 a known inhibitor of the PI3K [30]. Cells were treated with $50 \mu \mathrm{M}$ LY294002 for one hour min. Cells were then washed and lysed in SDS-PAGE sample buffer (Tris $300 \mathrm{mM}$ [pH 8.9], SDS 5\% (W/V), glycerol 20\% (V/V) (all purchased from Euromedex, Souffelweyersheim, France), $\beta$-mercaptoethanol $750 \mathrm{mM}$ and bromophenol blue (Thermo Fisher Scientific) for western blot analysis.

\section{5. siRNA, eV and $v R N F 41$ Transfection}

For down expression of RNF41, three days after induction of differentiation, OIS cells were transfected with Stealth RNAi Negative Control or siRNF41 duplexes using Lipofectamine RNAiMAX Transfection Reagent (all from Invitrogen, Thermo Fisher Scientific) according to manufacturer's instructions. After 12-h incubation in transfection medium, cells were treated or not with IFNI, polyIC and palmitate as indicated in the figures legends. For RNF41 overexpression, three days after induction of differentiation, OIR cells were transfected with $250 \mathrm{ng}$ of untagged cDNA cloned (NM_ 005785) in PCMV6-XL4 vector (vRNF41) or with empty PCMV6-XL4 vector (eV) (OriGene, Rockville, USA) mixed with turbofectin (OriGene) according to manufacturer's instructions. After 6-h incubation in transfection medium, cells were treated or not with palmitate and insulin as described above.

\subsection{Western Blot}

Total proteins were fractionated by SDS-PAGE and transferred electrophoretically to nitrocellulose membranes. Membranes were then incubated in Odyssey Blocking Buffer (LI-COR Biosciences, Eurobio, Les Ulis, France) for $45 \mathrm{~min}$ before addition of specific antibodies: Phospho-Akt (Ser473), Akt, Phospho-AS160 (Thr642) and AS160 antibodies (Cell Signaling Technology, Ozyme, Saint-Quentin-en-Yvelines), RNF41 antibody (Bethyl Laboratories, Euromedex), Actin-pan (Cell Signaling Technology) and species-directed secondary antibodies conjugated to IRDye800 or IRDye700 (Rockland Immunochemicals, tebubio, Le Perray-en-Yvelines, France), and analyzed with the Odyssey CLx Imaging System (LI-COR Biosciences, Bad Homburg, Germany). RNF41 protein quantification of each sample was corrected by the quantification of the corresponding loading control (Actin-pan), analyzed on the same membrane. Levels of protein expression were quantified using ImageJ software (National Institutes of Health, USA, available online at http://rsbweb.nih.gov/ij/index.html). a

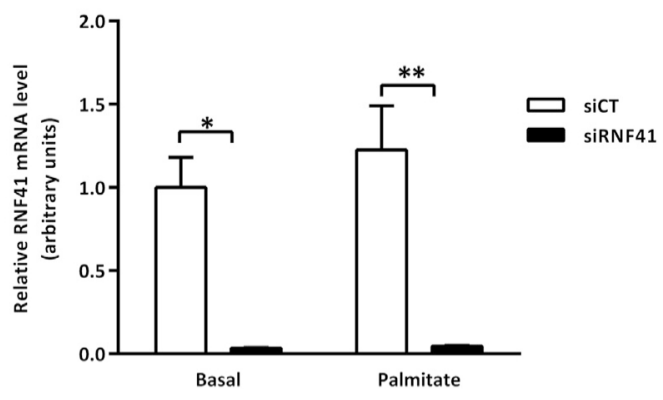

C

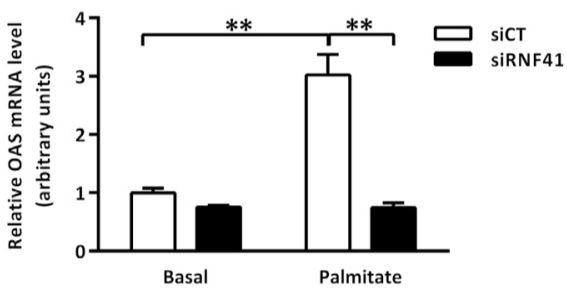

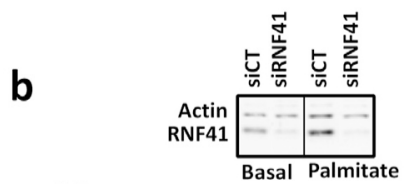
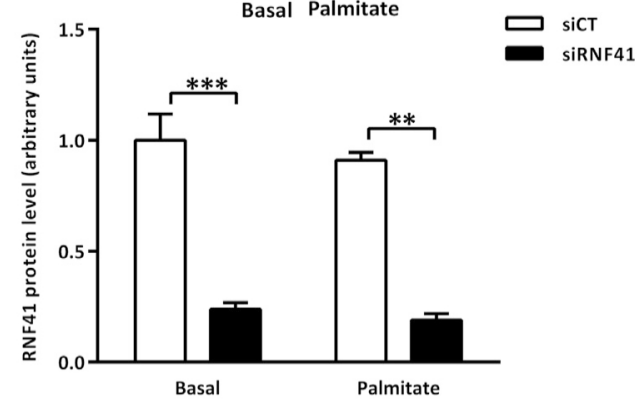

d

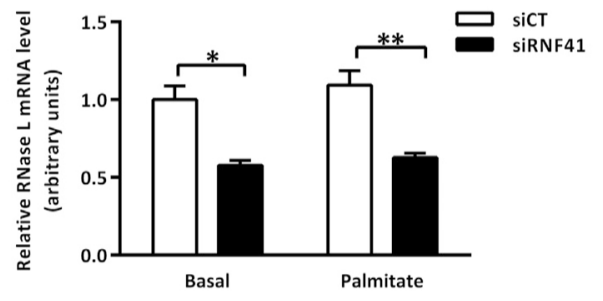

Fig. 3. Down regulation of RNF41 expression in OIS myotubes transfected with siRNF41. Panel a: Quantification by qPCR of RNF41 mRNA expression in OIS myotubes transfected with a control siRNA ( $\mathrm{SiCT}$ ) or a specific siRNA directed against RNF41 (siRNF41) and treated or not with palmitate. Data are expressed relative to the mean level of expression in siCT-transfected OIS myotubes in basal condition, which was set at 1. Error bars refer to the SEM obtained in three independent experiments. Group comparisons were performed using 2-way ANOVA and Tukey's multiple comparisons test. ${ }^{*} P_{\text {SiCT vs siRNF41 }}=0.02$ and ${ }^{* *} P_{\text {SiCT Palm vs siRNF41 Palm }}=0.008$. Panel b: Expression of RNF41 protein was assessed on total cellular extracts from OIS myotubes transfected with a siCT or siRNF41 and treated or not with palmitate by western blotting. Representative membrane scans are shown. Data are expressed relative to the mean level of expression in siCT-transfected OIS myotubes in basal condition, which was set at 1. Error bars refer to the SEM obtained in three independent experiments. Group comparisons were performed using 2-way ANOVA and Tukey's multiple comparisons test. ${ }^{* * *} P_{\text {SiCT vs siRNF41 }}=0.0008$ and ${ }^{* *} P_{\text {siCT Palm vs siRNF41 Palm }}=0.001$. Panel c: Quantification by qPCR of OAS mRNA expression in OIS myotubes transfected with siCT or siRNF41 and treated or not with palmitate. For each mRNA, data are expressed relative to the mean level of expression in siCT-transfected OIS myotubes in basal condition, which was set at 1 . Error bars refer to the SEM obtained in three independent experiments. Group comparisons were performed using 2-way ANOVA and Tukey's multiple comparisons test. OAS mRNA: ${ }^{* *} P_{\text {SiCT vs siCT Palm }}=0.003$ and ${ }^{* *} P_{\text {SiCT Palm vs siRNF41 Palm }}=0.001$. Panel d: Quantification by qPCR of RNase $L$ mRNA expression in OIS myotubes transfected with siCT or siRNF41 and treated or not with palmitate. For each mRNA, data are expressed relative to the mean level of expression in siCT-transfected OIS myotubes in basal condition, which was set at 1. Error bars refer to the SEM obtained in three independent experiments. Group comparisons were performed using 2-way ANOVA and Tukey's multiple comparisons test. RNase $L$ mRNA: ${ }^{*} P_{\text {SiCT vs siRNF41 }}=0.01$ and ${ }^{* *} P_{\text {SiCT Palm vs siRNF41 Palm }}=0.009$. 
2.7. RNA Purification, cDNA Synthesis and Quantitative Polymerase Chain Reaction ( $q P C R)$

Total RNA was isolated from differentiated SM cell progenitors (myotubes) with TRIzol (Invitrogen) and from SM biopsies (50 mg) of eight OIS and eight OIR subjects using RNeasy Fibrous Tissue Mini Kit (Qiagen, Courtabœuf, France). cDNA was generated from $1 \mu \mathrm{g}$ RNA by reverse transcription using the Verso cDNA Kit (Thermo Fisher Scientific). cDNA amplification was then performed by qPCR using the LightCycler 480 SYBR Green I Master (Roche Diagnostics, Meylan, France) and specific primers for sequences of interest (i.e. EEF1 $\alpha$ : forward CATGTGTGTTGAGAGCTTC, reverse GAAAACCAAAGTGGTCCAC; OAS1: forward AGTCATAAACTACCAGCAACTCT, reverse GGTCTCCAC CACCCAAG; RNase L: forward GCTCAAAGTAATGAAGAGGTGG, reverse ACATTCCGAAGCGTCCTA; RNF41: forward CTGTGTTCGGCTGTAGT, reverse CAGGTGCTTAATGCAGTTATG (Eurofins Scientific, Ebersberg, Germany). Relative mRNA expression was quantified according to the comparative cycle threshold method [31], using Ct values in the formula $2^{[\mathrm{Ct} \text { target gene-Ct reference gene] }}\left(2^{-\Delta \mathrm{Ct}}\right)$ with $e E F 1 \alpha$ (eukaryotic translation elongation factor 1 alpha) as stable reference gene [32,33].

\subsection{Statistical Analyses}

All group comparisons were performed using 2-way ANOVA followed by Tukey's post-hoc test for multiple comparisons. Mann-Whitney tests were performed for comparison of OIS and OIR SM biopsies. All analyses were performed with GraphPad PRISM 6 software. $P$ values are stated in the legends of the figures. For all tests, we considered that significance was reached when $P \leq 0.05$ with ${ }^{*} P \leq 0.05,{ }^{* *} P \leq 0.01$, ${ }^{* * *} P \leq 0.001$ and ${ }^{* * *} P \leq 0.0001$.

\section{Results}

\subsection{TRIF-IFNI Pathway Activation is Altered in OIR SM}

TRIF-IFNI pathway activation leads to increased expression of numerous ISG as ISG15, interferon responsive factor (IRF), Interferoninduced GTP-binding protein $M X 1$, double stranded activated protein kinase (PKR), OAS [34]. However, as we previously showed that OASRNase L pathway allows maintaining insulin sensitivity in palmitatetreated human myotubes [17], we analyzed OAS and RNase L mRNA expression in OIS and OIR SM biopsies. RNase $L$ mRNA expression was lower in OIR SM and OAS mRNA expression was similar in OIS and OIR SM (Fig. 1a), indicating a defect in the activation of TRIF-IFNI and OASRNase L pathways in OIR SM. To better understand the regulation of the TRIF-IFNI pathway in SM during obesity, we isolated and differentiated primary human SM cell progenitors from OIS and OIR SM biopsies [5]. SM cell progenitors represent an inestimable tool as once differentiated in myotubes they reproduce in vivo SM properties and maintain in culture the metabolic characteristics of the donor [35]. In fact, several studies have shown that the myotubes from T2D or insulin resistant donors show primary defects in insulin action [36] as insulin-stimulated phosphorylation of Akt [37], activation of Akt substrate of $160 \mathrm{kDa}$ (AS160) [38] even after several passages [27,35]. We first measured insulin response in OIS and OIR myotubes in basal condition and after treatment with palmitate in order to mimic TLR4 activation by NEFA in vivo [39]. We measured the phosphorylation of Akt and AS160, the a

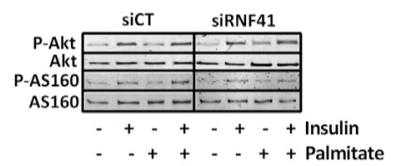

b

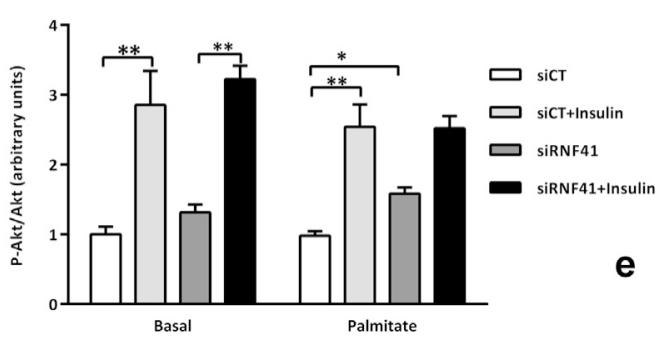

C

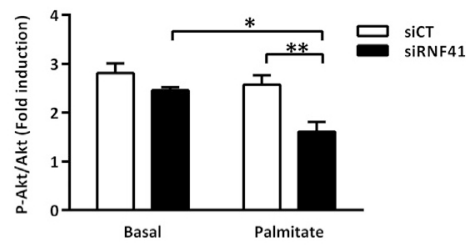

d
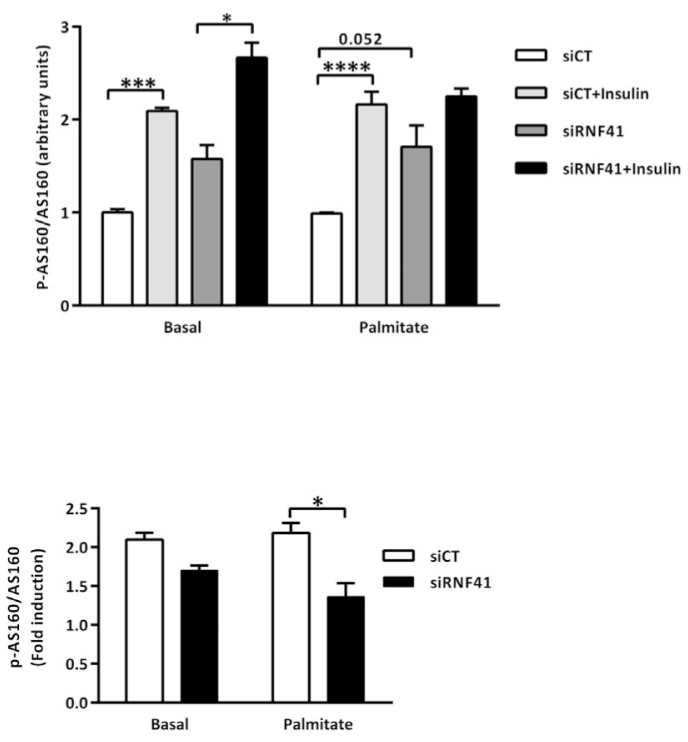

Fig. 4. Insulin response in OIS myotubes transfected with siRNF41. Panel a: Representative membrane scans for Phospho-Akt (Ser473), Phospho-AS160 (Thr642), Akt and AS160 protein expression are shown for OIS myotubes transfected with siCT or siRNF41, treated or not with palmitate and then stimulated or not with insulin. Panel b: OIS myotubes were transfected with siCT or siRNF41, treated or not with palmitate and then stimulated or not with insulin. To measure insulin response, Phospho-Akt (Ser473) and Akt protein expression was assessed on total cellular extracts by western blotting. Data are expressed relative to the mean level of Phospho-Akt (Ser473)/Akt ratio in siCT-transfected OIS myotubes in basal condition (with neither palmitate treatment nor insulin stimulation), which was set at 1. Error bars refer to the SEM obtained in three independent experiments. Group comparisons were performed using 2-way ANOVA and Tukey's multiple comparisons test. ${ }^{* *} P_{\text {SiCT vs siCT+ins }}={ }^{* *} P_{\text {siRNF41 vs siRNF41+ins }}=0.001$ and ${ }^{* *} P_{\text {siCT Palm vs siCT Palm+ins }}=0.007,{ }^{*} P_{\text {siCT Palm vs siRNF41 Palm }}=0.03$. Panel c: Insulin response is determined by the fold induction of Phospho-Akt (Ser473)/Akt ratio by insulin stimulation in basal condition or after palmitate treatment. Error bars refer to the SEM obtained in three independent experiments. Group comparisons were performed using 2 -way ANOVA and Tukey's multiple comparisons test. ${ }^{*} P_{\text {siRNF41 vs siRNF41 Palm }}=0.02$ and ${ }^{* *} P_{\text {siCT Palm vs siRNF41 }}$ Palm $=0.006$. Panel d: OIS myotubes were transfected with siCT or siRNF41, treated or not with palmitate and then stimulated or not with insulin. Phospho-AS160 (Thr642) and AS160 protein expression was assessed on total cellular extracts by western blotting. Data are expressed relative to the mean level of Phospho-AS160 (Thr642)/AS160 ratio in siCTtransfected OIS myotubes in basal condition (with neither palmitate treatment nor insulin stimulation), which was set at 1. Error bars refer to the SEM obtained in three independent experiments. Group comparisons were performed using 2-way ANOVA and Tukey's multiple comparisons test. ${ }^{* * *} P_{\text {SiCT vs siCT+ins }}=0.0001,{ }^{*} P_{\text {siRNF41 vs siRNF41+ins }}=0.02$ and ${ }^{* * * *} P_{\text {SiCT Palm }}$ vs sict Palm+ins < 0.0001. Panel e: Insulin response is determined by the fold induction of Phospho-AS160 (Thr642)/AS160 ratio by insulin stimulation in basal condition or after palmitate treatment. Error bars refer to the SEM obtained in three independent experiments. Group comparisons were performed using 2-way ANOVA and Tukey's multiple comparisons test. ${ }^{*} P_{\text {SiCT Palm vs siRNF41 Palm }}=0.02$. 
latter being a downstream effector of Akt allowing to appreciate GLUT4 trafficking $[38,40]$. In basal condition, insulin induced an increase of PAkt/Akt (Fig. 1b and c); and P-AS160/AS160 (Fig. 1b and e) ratio in OIS and OIR myotubes. However, the fold induction of P-Akt/Akt and P-AS160/AS160 ratio was lower in OIR myotubes, indicating a defect in insulin response compared to OIS myotubes (Fig. 1d and f). Moreover, while palmitate treatment nearly abolished insulin-stimulated increase of P-Akt/Akt and P-AS160/AS160 ratio in OIR myotubes, this was maintained in OIS myotubes (Fig. 1c and e), resulting in a lower fold induction of P-Akt/Akt and P-AS160/AS160 levels in OIR myotubes compared to OIS myotubes (Fig. 1d and f). Of note, reminiscent of our previous observation in OIS and OIR SM biopsies [5], P-Akt/Akt ratio level before insulin stimulation was higher in OIR myotubes compared to OIS myotubes (Fig. 1b and c) as was P-AS160/AS160 ratio level (Fig. 1e). In order to confirm that this effect on basal P-Akt is insulin dependent, we treated OIR myotubes with a PI3K inhibitor, i.e. Ly294002 [30]. Fig. 1g, illustrate the important decrease in P-Akt/Akt level in OIR myotubes incubated with Ly294002, thus demonstrating that the alteration in basal phosphorylation is indeed due to activation of insulin PI3K pathway. Analysis of ISG expression showed that OAS mRNA basal level was similar in OIS and OIR myotubes, but increased by palmitate treatment only in OIS myotubes (Fig. 2a). On the other hand, OIR myotubes expressed lower levels of RNase $L$ mRNA compared to OIS myotubes, regardless if they were treated or not with palmitate (Fig. 2b). Interestingly, OIS and OIR myotubes presented the same expression profile of OAS and RNase $L$ mRNA than OIS and OIR SM (compare Fig. 2a and b with Fig. 1a). Taking together these results suggest that TRIF-IFNI pathway following TLR4 activation is impaired in OIR myotubes.

\subsection{RNF41 Expression is Down-regulated in OIR SM and Myotubes}

As the E3 ubiquitin ligase RNF41 is a positive regulator of the TRIFIFNI pathway [21], we measured expression levels of RNF41 mRNA and protein in OIS and OIR SM. We observed no significant difference in RNF41 mRNA expression between OIS and OIR SM (Fig. 2c); however, RNF41 protein level was significantly lower in OIR compared to OIS SM (Fig. 2d). RNF41 mRNA was differentially expressed in OIS and OIR myotubes, regardless of palmitate treatment (Fig. 2e). As observed ex vivo, RNF41 protein level was lower in OIR myotubes compared to basal or palmitate-treated OIS myotubes (Fig. 2f).

\subsection{Regulation of RNF41 Expression Modulates Insulin Response in OIS and OIR Myotubes}

To investigate the role of RNF41 in the regulation of insulin response and OAS-RNase L pathway, we down-regulated its expression in OIS myotubes using a specific siRNA (siRNF41). siRNF41 highly reduced RNF41 mRNA and protein expression both in non-treated and palmitate-treated cells (Fig. 3a and b). Analysis of the OAS-RNase L pathway showed that OAS mRNA level increased following palmitate exposure in OIS myotubes transfected with a scrambled/control siRNA (siCT) and not with siRNF41 (Fig. 3c). RNase $L$ mRNA expression was lower in cells transfected with siRNF41 compared to cells transfected with siCT, regardless of palmitate treatment (Fig. 3d). Regarding the insulin response, insulin-stimulated P-Akt/Akt and P-AS160/AS160 ratio increased in cells transfected with siCT, both in basal condition and after palmitate treatment whereas siRNF41 transfection attenuated insulin effect in cells treated with palmitate (Fig. 4a, b and d). This was confirmed by a reduced fold induction of P-Akt/Akt and P-AS160/ AS160 in OIS myotubes transfected with siRNF41 compared to cells transfected with siCT, in palmitate-treated conditions (Fig. 4c and e). siRNF41 transfection induced an increase in P-Akt/Akt and P-AS160/ AS160 ratio after palmitate treatment despite no insulin stimulation (Fig. 4b and d), leading to the decrease of insulin effect on P-Akt/Akt and P-AS160/AS160 ratio (Fig. $4 \mathrm{~b}$ and d).

Reciprocally, we transfected OIR myotubes with a RNF41 expressing vector. We observed a small but significant increase of RNF41 protein expression only in cells not treated with palmitate and failed to observe such increase in OIR cells treated with palmitate (Supplementary Fig. 1a). However, this small RNF41 increase was sufficient to allow a better insulin response as attested by an increase of P-Akt/Akt and P-AS160/AS160 ratio fold induction (Supplementary Fig. 1c and d). We also observed an increase in OAS mRNA expression without modification of RNase $L$ mRNA expression (Supplementary Fig. 1b). Interestingly, when RNF41 expression is increased we observed a decrease in basal P-Akt/Akt ratio (Supplementary Fig. 1c). a

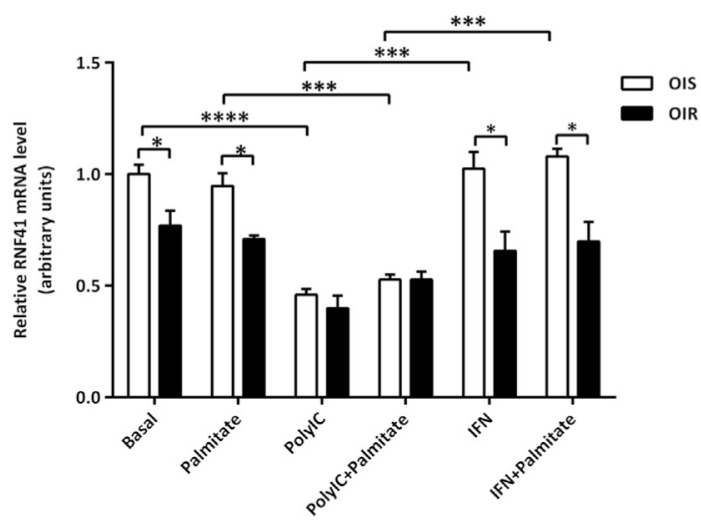

b
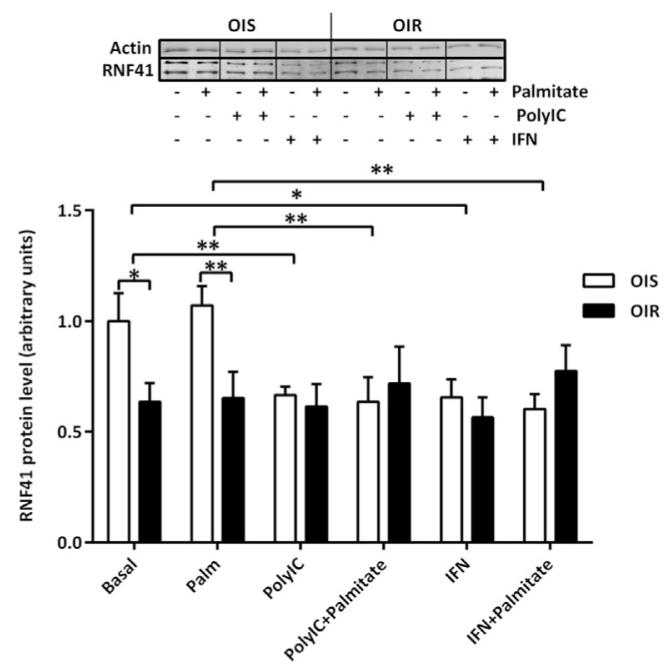

Fig. 7. RNF41 mRNA and protein expression in OIS and OIR myotubes treated with polyIC or IFNI. Panel a: Quantification by qPCR of RNF41 mRNA in OIS and OIR myotubes treated or not with palmitate and/or polyIC or IFNI. Data are expressed relative to the mean level of expression in OIS myotubes in basal condition, which was set at 1 . Error bars refer to the SEM obtained in three independent experiments. Group comparisons were performed using 2 -way ANOVA and Tukey's multiple comparisons test. ${ }^{*} P_{\text {OIS vs }}$ OIR $={ }^{*} P_{\text {OIS Palm vs } \text { OIR Palm }}=0.02$, ${ }^{* * * *} P_{\text {OIS vs }}$

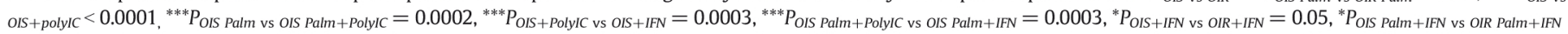
$=0.04$. Panel b: Expression of RNF41 protein was assessed on total cellular extracts from OIS and OIR myotubes treated or not with palmitate and/or polyIC or IFNI by western blotting. Representative membrane scans are shown. Data are expressed relative to the mean level of expression in OIS myotubes in basal condition, which was set at 1 . Error bars refer to the SEM obtained in three independent experiments. Group comparisons were performed using 2-way ANOVA and Tukey's multiple comparisons test. ${ }^{*} P_{\text {OIS }}$ vs oIR $=0.01$, ${ }^{* *} P_{\text {OIS }}$ Palm vs OIR Palm $=0.007,{ }^{* *} P_{\text {OIS vs OIS }+ \text { PolylC }}=0.009,{ }^{* *} P_{\text {OIS Palm vs OIS Palm }+ \text { PolyIC }}=0.002,{ }^{*} P_{\text {OIS vs OIS }+I F N}=0.02,{ }^{* *} P_{\text {OIS Palm vs OIS Palm+IFN }}=0.006$. 


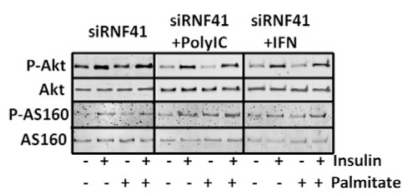

b

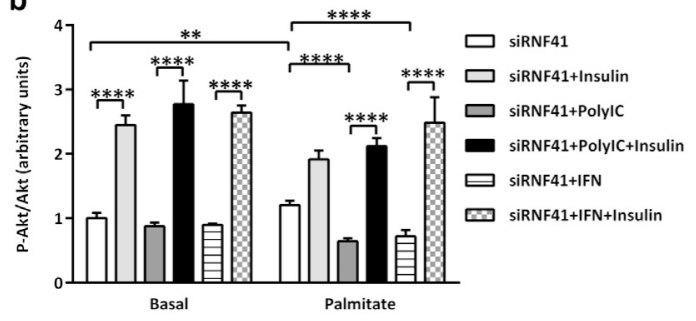

C

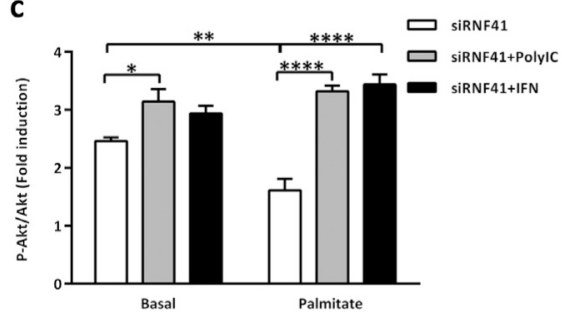

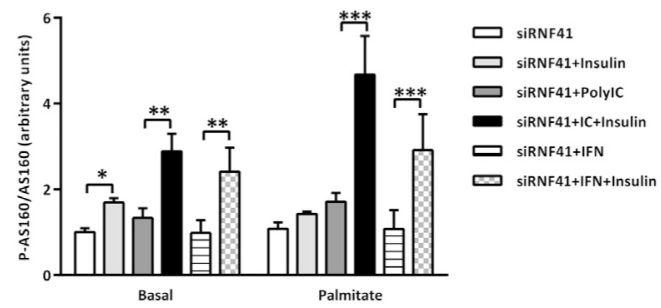

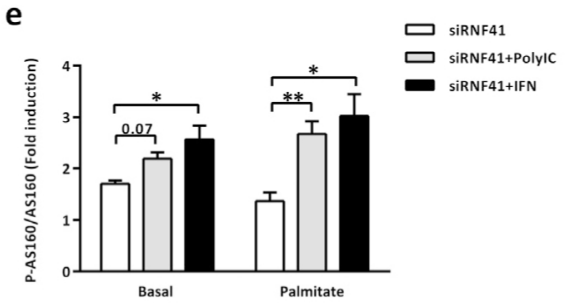

Fig. 8. PolyIC and IFNI treatments improve insulin response in OIS myotubes transfected with siRNF41. Panel a: Representative membrane scans for Phospho-Akt (Ser473), Phospho-AS160 (Thr642), Akt and AS160 protein expression are shown for OIS myotubes transfected with siRNF41 and treated or not with palmitate, and/or polyIC or IFNI, and then stimulated or not with insulin. Panel b: To measure insulin response, Phospho-Akt (Ser473) and Akt protein expression was assessed on total cellular extracts by western blotting. Data are expressed relative to the mean level of Phospho-Akt (Ser473)/Akt ratio in siRNF41-transfected OIS myotubes in basal condition (non-treated and not stimulated with insulin), which was set at 1. Error bars refer to the SEM obtained in three independent experiments. Group comparisons were performed using 2-way ANOVA and Tukey's multiple comparisons test. ${ }^{* * * *} P_{\text {siRNF41 vs siRNF41+ins }}$ $={ }^{* * * *} P_{\text {siRNF } 41+\text { PolylC vs siRNF41+PolyIC }+ \text { ins }}={ }^{* * * *} P_{\text {siRNF } 41+I F N \text { vs siRNF41+IFN+ins }}={ }^{* * * *} P_{\text {siRNF41 Palm }+ \text { PolyIC vs siRNF41 Palm }+ \text { PolyIC+ins }}={ }^{* * * * *} P_{\text {siRNF41 Palm+IFNI vs siRNF41 Palm+IFNI+ins }}={ }^{* * * *} P_{\text {siRNF41 Palm vs }}$ siRNF41 Palm+PolyIC $={ }^{* * * *} P_{\text {SiRNF41 Palm vs siRNF41Palm }+I F N}<0.0001$ and ${ }^{* *} P_{\text {SiRNF41 vs siRNF41 Palm }}=0.009$ Panel c: Data are expressed relative to the fold induction of Phospho-Akt (Ser473)/Akt ratio in basal condition or with palmitate and/or polyIC or IFNI treatment. Error bars refer to the SEM obtained in three independent experiments. Group comparisons were performed using 2-way ANOVA and Tukey's multiple comparisons test. ${ }^{*} P_{\text {siRNF41 vs siRNF41 +PolylC }}=0.04,{ }^{* *} P_{\text {siRNF41 vs siRNF41 Palm }}=0.009$, ${ }^{* * * *} P_{\text {siRNF41 Palm vs siRNF41 Palm }+ \text { PolylC }}={ }^{* * * *} P_{\text {siRNF41 Palm vs }}$ siRNF41Palm+IFN < 0.0001. Panels d: Phospho-AS160 (Thr642) and AS160 protein expression was assessed on total cellular extracts by western blotting. Data are expressed relative to the mean level of Phospho-AS160 (Thr642)/AS160 ratio in siRNF41-transfected OIS myotubes in basal condition (non-treated and not stimulated with insulin), which was set at 1. Error bars refer to the SEM obtained in three independent experiments. Group comparisons were performed using 2-way ANOVA and Tukey's multiple comparisons test. ${ }^{*} P_{\text {siRNF4I }}$ vs

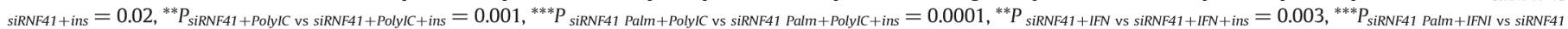
Palm+IFNI+ins $=0.0001$. Panel e: Data are expressed relative to the fold induction of Phospho-AS160 (Thr642)/AS160 ratio in basal condition or with palmitate and/or polyIC or IFNI treatment. Error bars refer to the SEM obtained in three independent experiments. Group comparisons were performed using 2-way ANOVA and Tukey's multiple comparisons test. ${ }^{*} P_{\text {SiRNF41 Palm vs siRNF41 Palm+PolyIC }}=0.007,{ }^{*} P_{\text {SiRNF41 Palm vs siRNF41Palm }+ \text { IFN }}=0.01,{ }^{*} P_{\text {SiRNF41 vs siRNF41+IFN }}=0.01$.

\subsection{IFNI and polyIC Treatments Restore Insulin Sensitivity in OIR Myotubes}

Our current results indicated that, in myotubes, IR induced by palmitate exposure is associated with reduced RNF41 level and reduced activation of TRIF-IFNI signaling pathway following TLR4 activation. Therefore, in order to bypass TLR4 activation and directly induce ISG mRNA expression, we treated both OIS and OIR myotubes with IFNI or polyIC a potent inductor of IFNI and ISG via TLR3 $[10,11,41]$. PolyIC and IFNI treatments induced a robust increase in OAS mRNA expression in OIS and OIR myotubes (Fig. 5a). Interestingly, RNase $L$ mRNA expression was induced by polyIC in OIR myotubes only, thus leading to similar levels of RNase $L$ mRNA in OIS and OIR myotubes independently of palmitate treatment. RNase $L$ mRNA was not modulated by IFNI treatment in OIS myotubes, and only poorly modulated in OIR myotubes. Nevertheless, this modest increase was sufficient to cancel the difference of RNase $L$ mRNA expression between OIR and OIS myotubes (Fig. 5b).

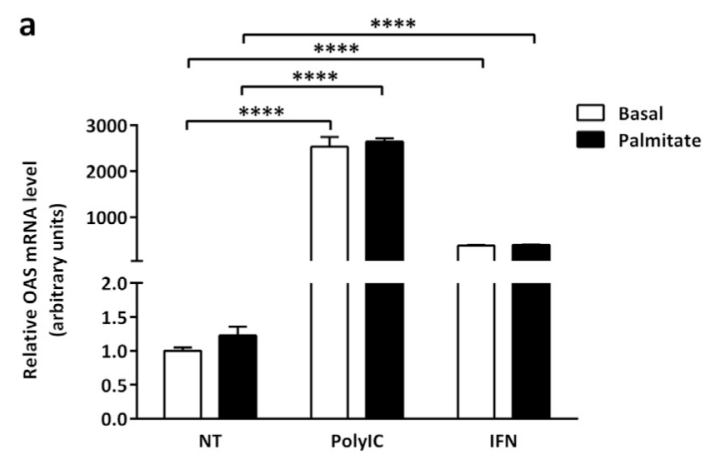

b

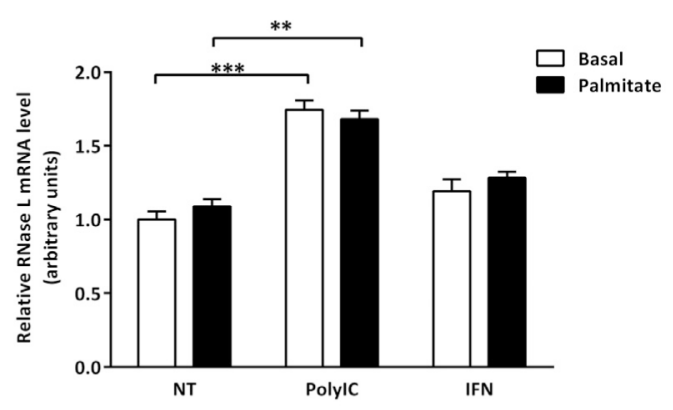

Fig. 9. PolyIC and IFNI treatments rescue ISG mRNA expression in OIS myotubes transfected with siRNF41. Quantification by qPCR of OAS mRNA (Panel a), and RNase L mRNA (Panel b) in OIS myotubes transfected with siRNF41 and treated or not with palmitate and/or polyIC or IFNI (NT: not treated). Data are expressed relative to the mean level of expression in OIS myotubes in basal condition not treated with polylC or IFN, which was set at 1. Error bars refer to the SEM obtained in three independent experiments. Group comparisons were performed using 2-way ANOVA and Tukey's multiple comparisons test. OAS mRNA: all ${ }^{* * *} P<0.0001$. RNase L mRNA: ${ }^{* * *} P_{\text {SiRNF41 vs siRnF41+PolylC }}=0.0003,{ }^{* *} P_{\text {siRnF41 Palm vs siRnF41 Palm+PolylC }}=0.001$ 
PolyIC and IFNI treatments had no effect on insulin-stimulated increase of P-Akt/Akt or P-AS160/AS160 ratio in OIS myotubes; however, both restored insulin response in OIR palmitate-treated myotubes (Fig. 6a, b and d), leading to an increase in fold induction of P-Akt/Akt and P-AS160/AS160 ratio (Fig. $6 \mathrm{c}$ and e). Interestingly, the effect of polyIC and IFNI exposure was mainly due to a decrease in P-Akt/Akt ratio in non-insulin-stimulated and palmitate-treated OIR myotubes (Fig. 6a and b).

Regarding RNF41 expression, in OIS myotubes, polyIC and IFNI treatments induced a down-regulation of RNF41 protein expression (Fig. 7b) while only polyIC induced a down-regulation of RNF41 mRNA expression (Fig. 7a). However, neither polyIC nor IFNI treatment modulated RNF41 mRNA or protein expression in OIR myotubes (Fig. 7a and b).

3.5. IFNI and polyIC Treatments Rescue siRNF41-induced IR in OIS Myotubes

As IFNI and polyIC treatments allowed restoring insulin response in OIR myotubes, we investigated the effect of such treatments in OIS myotubes previously transfected with siRNF41 to induce IR following palmitate treatment. PolyIC and IFNI exposure induced an increase in insulin response in siRNF41-transfected OIS myotubes treated with palmitate (Fig. 8a, b and d), leading to increased fold induction of P-Akt/Akt (Fig. 8c) and P-AS160/AS160 ratio (Fig. 8e). Again, polyIC and IFNI exposure led to a decrease in P-Akt/Akt ratio in non-insulin-stimulated and palmitate-treated OIS myotubes (Fig. 8a, b). As described above, OAS mRNA was not induced by palmitate treatment and RNase $L$ mRNA expression was down-regulated in OIS myotubes transfected with siRNF41 (Fig. 5a and b). PolyIC and IFNI treatment strongly induced OAS mRNA in cells treated or not with palmitate (Fig. 9a); RNase L mRNA was induced only by polyIC treatment (Fig. 9b).

\section{Discussion}

Several studies have shown the role of TLR4-mediated MyD88 activation in IR development [12]. However, TLR4 signaling involves a second pathway: the TRIF-IFNI-dependent pathway. In this study, we aimed to analyze the regulation by RNF41 of TRIF-IFNI pathway and insulin response in OIS and OIR SM. In agreement with other studies [12], we previously showed that IkB expression is lower in OIR compared to OIS SM, suggesting that MyD88 pathway is more activated in OIR SM [5]. Here, we show that RNase L mRNA expression is lower in OIR compared to OIS SM whereas OAS mRNA level is similar, indicating a defect in the activation of TRIF-IFNI and OAS-RNase L pathways in OIR SM.

Based on the identification of RNF41 as a central regulator of the balance between MyD88 and TRIF-IFNI pathways [21] and the observation that RNF41 is expressed at a lower level in OIR SM, we hypothesized that the preferential activation of MyD88 pathway in OIR SM could be due to lower RNF41 expression. To investigate this hypothesis, we used primary human SM cell progenitors purified from human SM biopsies and differentiated in myotubes to modulate the expression of genes of interest. In fact, several studies have shown that myotubes display morphological, metabolic and biochemical similarities to adult skeletal muscle $[42,43]$. Admittedly, a combination of genetic and epigenetic mechanisms is involved in maintaining in vitro these in vivo characteristics $[44,45]$. Myotubes from T2D or insulin resistant donors show primary defects in glucose transport and glycogen synthase activity [43,46,47] insulin-stimulated phosphorylation of Akt [37], activation of Akt substrate of $160 \mathrm{kDa}$ (AS160) [38], lipolysis and insulin action [36] even after several passages $[27,35]$. SM cell progenitors thus offer a unique model to study insulin sensitivity/resistance. We purified SM cell progenitors from OIS and OIR women, extensively characterized for their insulin sensitivity at systemic and tissue level [5]. OIR myotubes are more sensitive than OIS myotubes to an inflammatory stress such as palmitate exposure. In fact, palmitate treatment led to the decrease of insulin-stimulated fold induction of P-Akt/Ak and P-AS160/AS160 ratio in OIR myotubes while it did not modify insulin response of OIS myotubes. To investigate the molecular mechanism behind TRIF-IFNI pathway activation, we focused on OAS-RNase L pathway as we and others previously reported its role in energy metabolism and obesity $[17,33,48,49]$. OIR myotubes were characterized by low level of RNase $L$ mRNA, by an absence of increase in OAS mRNA expression following palmitate treatment and by decreased level of RNF41 expression compared to OIS myotubes. However, treatment of OIR myotubes with IFNI or with polyIC, increased insulin-stimulated fold induction of P-Akt/Akt and P-AS160/AS160 ratio and restored OAS and RNase $L$ mRNA expression, indicating that TRIF-IFNI and IFNI pathways are functional in OIR myotubes. The absence of a positive induction of OAS mRNA expression in palmitate-treated OIR myotubes could thus be the consequence of a defect in TRIF-IFNI pathway activation at the expense of MyD88 pathway activation downstream of TLR4. Such defect could be due to lower level of RNF41 in OIR myotubes compared to OIS myotubes. In fact, when OIS myotubes were transfected with siRNF41, RNase $L$ mRNA expression was down-regulated, palmitate exposure did not induce OAS mRNA expression and inhibited insulin response. The inhibition of RNF41 expression thus mimicked OIR phenotype in OIS myotubes. At the opposite, transfection of OIR myotubes with a RNF41 vector lead to an increase in P-Akt/Akt and P-AS160/AS160 fold induction attesting of an improvement in insulin response. However, this overexpression of RNF41 was associated with OAS mRNA increase and no modification of RNase $L$ mRNA levels. Interestingly we can only obtain a modest increase in RNF41 expression in OIR myotubes not treated with palmitate. This result is indicative of an important regulation of RNF41 expression in OIR myotubes particularly during palmitate treatment. IFNI and polyIC treatments of siRNF41-transfected OIS myotubes allowed to by-pass TLR4 activation and, as in OIR myotubes, restored insulin response and palmitate-induced OAS mRNA expression. This set of data confirms the central role of OAS-RNase L pathway in insulin response $[17,33,48]$. In agreement with the previous identified role of RNF41, the down-regulation of its expression with siRNF41 did not block the increase in OAS and RNase L expression induced by polyIC or IFNI. In fact, RNF41 acts downstream to TLR4 and upstream to IFNI and ISG expression [21]. Wang et al. previously showed that polyIC treatment of mice peritoneal macrophages or of the macrophage cell line RAW264.7 up-regulates RNF41 expression [21], when we on the other hand, observed that polyIC and IFNI treatments down-regulated RNF41 expression in OIS myotubes. We speculated that the establishment of a negative feedback regulation of RNF41 levels by polyIC and IFNI could also limit the induction of IFNI and ISG expressions and therefore their potential deleterious effects due to a prolonged activation of the TRIF-IFNI and IFNI pathways [50]. Such discrepancy between our results and Wang's findings could be due to the difference in the experimental cell models/lines investigated. Indeed, the regulation of innate immunity and TRIF-IFNI pathway is known to be different not only between mammals (humans vs mice) but also between cell types/models/lines [50-52].

The decrease in insulin sensitivity in OIR myotubes was characterized by the increase of P-Akt/Akt ratio without insulin exposure. Such constitutive phosphorylation of Akt is due to the activation of the insulin PI3K pathway as its decrease is attested in the presence of the specific PI3K inhibitor Ly294002. Constitutive phosphorylation of Akt limits insulin response and has previously been observed in animal models of diet-induced obesity and IR [53-55] and in human cell models [55,56]. In these studies, the over-activation of Akt induced IR development. A maintained equilibrium in Akt phosphorylation/dephosphorylation at $\mathrm{Ser}^{473}$ is one of the central mechanisms controlling the insulin signaling pathway. Therefore, it is tightly regulated by several feedback pathways, themselves interconnected through Akt signaling [57,58]. Our actual data show that the treatment of OIR myotubes with IFNI or polyIC reduced basal Akt phosphorylation, resulting in increased insulin response. However, additional studies would be necessary to determine the role of IFNI in SM insulin response during obesity. 
In conclusion, we demonstrated that low RNF41 expression blunted TRIF-IFNI and OAS-RNase L signaling pathways, downstream to TLR4 activation. Consequently, insulin sensitivity is impaired in OIR myotubes. Our results thus show that RNF41 plays a pivotal role in the maintenance of insulin sensitivity in SM and that TLR4 activation is not necessarily deleterious and its negative effect on insulin sensitivity mainly depends on the downstream pathway that is activated. One strength of our study is that we explored the role of IFNI pathway in myotubes obtained from a homogenous group of OIS and OIR women highly characterized in vivo for their insulin sensitivity and inflammatory status [5]. However this study only concerned grade 1 obese women, as such complementary studies would be interesting to evaluate RNF41 and IFNI pathway regulation in grade 2 and 3 obese women and men. Nevertheless, the mechanisms involved in RNF41 regulation and the targets of the IFNI and OAS-RNase L pathways remain to be identified in order to improve our understanding of IR development in different states of obesity.

\section{Fundings}

This work was supported by grants from Institut National de la Santé et de la Recherche Médicale (INSERM), Université de Montpellier (UM), Centre National de la Recherche Scientifique (CNRS) and CHRU Montpellier PROM8685. CA and OF were both recipient of a Ministère de l'Enseignement Supérieur et de la Recherche (MESR) fellowship.

\section{Acknowledgments}

The authors gratefully acknowledge Marie Hokayem for the critical reading of the manuscript.

\section{Authors' contribution}

Conceived and designed the experiments: $\mathrm{CBi}$ and TS. Experimental investigations: $\mathrm{CA}, \mathrm{CBi}, \mathrm{CB}, \mathrm{OF}, \mathrm{KL}, \mathrm{PS}, \mathrm{AB}$. Recruitment of the volunteers and SM biopsies: AS and JM. Data analysis: $\mathrm{CBi}, \mathrm{CB}, \mathrm{CA}$. Wrote the manuscript: $\mathrm{CBi}$ and $\mathrm{OF}$. Review of the manuscript: all authors.

\section{Conflicts of interest}

None.

\section{References}

[1] Kahn BB, Flier JS. Obesity and insulin resistance. J Clin Invest 2000;106(4):473-81.

[2] Chow L, From A, Seaquist E. Skeletal muscle insulin resistance: the interplay of local lipid excess and mitochondrial dysfunction. Metabolism 2010;59(1):70-85.

[3] DeFronzo RA, Tripathy D. Skeletal muscle insulin resistance is the primary defect in type 2 diabetes. Diabetes Care 2009;32(Suppl. 2):S157-63.

[4] Tsatsoulis A, Mantzaris MD, Bellou S, et al. Insulin resistance: an adaptive mechanism becomes maladaptive in the current environment - an evolutionary perspective. Metabolism 2013;62(5):622-33.

[5] Amouzou C, Breuker C, Fabre O, et al. Skeletal muscle insulin resistance and absence of inflammation characterize insulin-resistant grade I obese women. PLoS One 2016; 11(4):e0154119.

[6] Gomes JMG, Costa JA, Alfenas RCG. Metabolic endotoxemia and diabetes mellitus: a systematic review. Metabolism 2017;68:133-44.

[7] Cuda C, Badawi A, Karmali M, et al. Polymorphisms in toll-like receptor 4 are associated with factors of the metabolic syndrome and modify the association between dietary saturated fat and fasting high-density lipoprotein cholesterol. Metabolism 2011;60(8):1131-5.

[8] Dasu MR, Jialal I. Free fatty acids in the presence of high glucose amplify monocyte inflammation via toll-like receptors. Am J Physiol Endocrinol Metab 2011;300(1):E145-4.

[9] Jialal I, Kaur H, Devaraj S. Toll-like receptor status in obesity and metabolic syndrome: a translational perspective. J Clin Endocrinol Metab 2014;99(1):39-48.

[10] Takeda K, Akira S. TLR signaling pathways. Semin Immunol 2004;16(1):3-9.

[11] Uematsu S, Akira S. Toll-like receptors and type I interferons. J Biol Chem 2007; 282(21):15319-23.
[12] Velloso LA, Folli F, Saad MJ. TLR4 at the crossroads of nutrients, gut microbiota and metabolic inflammation. Endocr Rev 2015;36(3):245-71

[13] Ivashkiv LB, Donlin LT. Regulation of type I interferon responses. Nat Rev Immunol 2014;14(1):36-49.

[14] Honda K, Taniguchi T. IRFs: master regulators of signalling by toll-like receptors and cytosolic pattern-recognition receptors. Nat Rev Immunol 2006;6(9):644-58.

[15] Bisbal C, Silverman RH. Diverse functions of RNase L and implications in pathology Biochimie 2007;89(6-7):789-98.

[16] Kariko K, Ni H, Capodici J, et al. mRNA is an endogenous ligand for toll-like receptor 3. J Biol Chem 2004;279(13):12542-50.

[17] Fabre O, Breuker C, Amouzou C, et al. Defects in TLR3 expression and RNase L activation lead to decreased MnSOD expression and insulin resistance in muscle cells of obese people. Cell Death Dis 2014;5:e1136.

[18] Bisbal C, Martinand C, Silhol M, et al. Cloning and characterization of a RNAse L inhibitor. A new component of the interferon-regulated 2-5A pathway. J Biol Chem 1995;270(22):13308-17.

[19] Karelis AD, Brochu M, Rabasa-Lhoret R. Can we identify metabolically healthy but obese individuals (MHO)? Diabetes Metab 2004;30(6):569-72.

[20] Bluher M. Are there still healthy obese patients? Curr Opin Endocrinol Diabetes Obes 2012;19(5):341-6.

[21] Wang C, Chen T, Zhang J, et al. The E3 ubiquitin ligase Nrdp1 'preferentially' promotes TLR-mediated production of type I interferon. Nat Immunol 2009;10(7): 744-52.

[22] Thomas C, Sirvent P, Perrey S, et al. Relationships between maximal muscle oxidative capacity and blood lactate removal after supramaximal exercise and fatigue indexes in humans. J Appl Physiol 2004;97(6):2132-8.

[23] Salehzada T, Cambier L, Vu Thi N, et al. Endoribonuclease L (RNase L) regulates the myogenic and adipogenic potential of myogenic cells. PLoS One 2009;4(10):e7563.

[24] Blau H, Webster C. Isolation and characterization of human muscle cells. Proc Natl Acad Sci U S A 1981;78(9):5623-7.

[25] Barro M, Carnac G, Flavier S, et al. Myoblasts from affected and non-affected FSHD muscles exhibit morphological differentiation defects. J Cell Mol Med 2010;14(12):275-89.

[26] Laurens C, Louche K, Sengenes C, et al. Adipogenic progenitors from obese human skeletal muscle give rise to functional white adipocytes that contribute to insulin resistance. Int J Obes (Lond) 2016;40(3):497-506.

[27] Nehlin JO, Just M, Rustan AC, et al. Human myotubes from myoblast cultures undergoing senescence exhibit defects in glucose and lipid metabolism. Biogerontology 2011;12(4):349-65.

[28] Wang X, Yu W, Nawaz A, et al. Palmitate induced insulin resistance by PKCthetadependent activation of mTOR/S6K pathway in $\mathrm{C} 2 \mathrm{C} 12$ myotubes. Exp Clin Endocrinol Diabetes 2010;118(9):657-61.

[29] Wang C, Liu M, Riojas RA, et al. Protein kinase C theta (PKCtheta)-dependent phosphorylation of PDK1 at Ser504 and Ser532 contributes to palmitate-induced insulin resistance. J Biol Chem 2009;284(4):2038-44.

[30] Vlahos C], Matter WF, Hui KY, et al. A specific inhibitor of phosphatidylinositol 3 kinase, 2-(4-morpholinyl)-8-phenyl-4H-1-benzopyran-4-one (LY294002). J Biol Chem 1994;269(7):5241-8.

[31] Schmittgen TD, Livak KJ. Analyzing real-time PCR data by the comparative C(T) method. Nat Protoc 2008;3(6):1101-8.

[32] Dostal DE, Rothblum KN, Baker KM. An improved method for absolute quantification of mRNA using multiplex polymerase chain reaction: determination of renin and angiotensinogen mRNA levels in various tissues. Anal Biochem 1994;223(2): 239-50.

[33] Fabre O, Salehzada T, Lambert K, et al. RNase L controls terminal adipocyte differentiation, lipids storage and insulin sensitivity via CHOP10 mRNA regulation. Cell Death Differ 2012;19(9):1470-81.

[34] Schneider WM, Chevillotte MD, Rice CM. Interferon-stimulated genes: a complex web of host defenses. Annu Rev Immunol 2014;32:513-45.

[35] Aas V, Bakke SS, Feng YZ, et al. Are cultured human myotubes far from home? Cell Tissue Res 2013;354:671-82.

[36] Kase ET, Feng YZ, Badin PM, et al. Primary defects in lipolysis and insulin action in skeletal muscle cells from type 2 diabetic individuals. Biochim Biophys Acta 2015; 1851(9):1194-201.

[37] Cozzone D, Frojdo S, Disse E, et al. Isoform-specific defects of insulin stimulation of Akt/protein kinase B (PKB) in skeletal muscle cells from type 2 diabetic patients. Diabetologia 2008;51(3):512-21.

[38] Vendelbo MH, Clasen BF, Treebak JT, et al. Insulin resistance after a 72-h fast is associated with impaired AS160 phosphorylation and accumulation of lipid and glycogen in human skeletal muscle. Am J Physiol Endocrinol Metab 2012;302(2): E190-00.

[39] Kim F, Pham M, Luttrell I, et al. Toll-like receptor-4 mediates vascular inflammation and insulin resistance in diet-induced obesity. Circ Res 2007;100(11):1589-96.

[40] Miinea CP, Sano H, Kane S, et al. AS160, the Akt substrate regulating GLUT4 translocation, has a functional Rab GTPase-activating protein domain. Biochem J 2005; 391(Pt 1):87-93.

[41] Field AK, Tytell AA, Lampson GP, et al. Inducers of interferon and host resistance. II. Multistranded synthetic polynucleotide complexes. Proc Natl Acad Sci U S A 1967; 58(3):1004-10.

[42] Gaster M, Kristensen SR, Beck-Nielsen H, et al. A cellular model system of differentiated human myotubes. Apmis 2001;109(11):735-44.

[43] Henry RR, Abrams L, Nikoulina S, et al. Insulin action and glucose metabolism in nondiabetic control and NIDDM subjects. Comparison using human skeletal muscle cell cultures. Diabetes 1995;44(8):936-46.

[44] Howlett KF, McGee SL. Epigenetic regulation of skeletal muscle metabolism. Clin Sc (Lond) 2016;130(13):1051-63. 
[45] Dos Santos JM, Moreli ML, Tewari S, et al. The effect of exercise on skeletal muscle glucose uptake in type 2 diabetes: an epigenetic perspective. Metabolism 2015; 64(12):1619-28.

[46] Gaster M, Petersen I, Hojlund K, et al. The diabetic phenotype is conserved in myotubes established from diabetic subjects: evidence for primary defects in glucose transport and glycogen synthase activity. Diabetes 2002;51(4): $921-7$.

[47] McIntyre EA, Halse R, Yeaman SJ, et al. Cultured muscle cells from insulin-resistant type 2 diabetes patients have impaired insulin, but normal 5-amino-4imidazolecarboxamide riboside-stimulated, glucose uptake. J Clin Endocrinol Metab 2004;89(7):3440-8.

[48] Wang Y, Chiang H, Huang Y, et al. A link between adipogenesis and innate immunity: RNase-L promotes 3T3-L1 adipogenesis by destabilizing Pref- 1 mRNA. Cell Death Dis 2016;7(11):e2458.

[49] Wang YT, Tseng PH, Chen CL, et al. Human serum RNase-L level is inversely associated with metabolic syndrome and age. Cardiovasc Diabetol 2017;16(1):46.

[50] Schreiber G. The molecular basis for differential type I interferon signaling. J Biol Chem 2017;292(18):7285-94.

[51] Mestas J, Hughes CCW. Of mice and not men: differences between mouse and human immunology. J Immunol 2004;172:2731-8.
[52] Schroder K, Irvine KM, Taylor MS, et al. Conservation and divergence in toll-like receptor 4-regulated gene expression in primary human versus mouse macrophages. Proc Natl Acad Sci U S A 2012;109(16):944-53.

[53] Buren J, Liu HX, Lauritz J, et al. High glucose and insulin in combination cause insulin receptor substrate- 1 and -2 depletion and protein kinase $B$ desensitisation in primary cultured rat adipocytes: possible implications for insulin resistance in type 2 diabetes. Eur J Endocrinol 2003;148:157-67.

[54] Liu HY, Hong T, Wen GB, et al. Increased basal level of Akt-dependent insulin signaling may be responsible for the development of insulin resistance. Am J Physiol Endocrinol Metab 2009;297(4):E898-06.

[55] Khamzina L, Veilleux A, Bergeron S, et al. Increased activation of the mammalian target of rapamycin pathway in liver and skeletal muscle of obese rats: possible involvement in obesity-linked insulin resistance. Endocrinology 2005;146(3):1473-81.

[56] Tremblay F, Gagnon A, Veilleux A, et al. Activation of the mammalian target of rapamycin pathway acutely inhibits insulin signaling to Akt and glucose transport in 3T3-L1 and human adipocytes. Endocrinology 2005;146(3):1328-37.

[57] Han EK, Leverson JD, McGonigal T, et al. Akt inhibitor A-443654 induces rapid Akt Ser-473 phosphorylation independent of mTORC1 inhibition. Oncogene 2007;26(38):5655-61.

[58] Risso G, Blaustein M, Pozzi B, et al. Akt/PKB: one kinase, many modifications. Biochem J 2015;468(2):2013-214 